Itinéraires Itinéraires

Littérature, textes, cultures

\title{
Stratégies discursives et processus de mémorisation dans le genre e-conférence
}

Florimond Rakotonoelina

\section{(2) OpenEdition}

\section{Journals}

Édition électronique

URL : http://journals.openedition.org/itineraires/181

DOI : 10.4000/itineraires.181

ISSN : 2427-920X

Éditeur

Pléiade

\section{Édition imprimée}

Date de publication : 1 juillet 2011

Pagination : 147-174

ISBN : 978-2-296-54673-8

ISSN : 2100-1340

Référence électronique

Florimond Rakotonoelina, « Stratégies discursives et processus de mémorisation dans le genre econférence », Itinéraires [En ligne], 2011-2 | 2011, mis en ligne le 03 février 2015, consulté le 30 avril 2019. URL : http://journals.openedition.org/itineraires/181; DOI : 10.4000/itineraires.181

\section{cc) (i) (9)}

Itinéraires est mis à disposition selon les termes de la licence Creative Commons Attribution - Pas d'Utilisation Commerciale - Pas de Modification 4.0 International. 


\title{
Stratégies discursives et processus de mémorisation dans le genre e-conférence
}

\begin{abstract}
This article examines the concept of memory explained by learning theories within the "e-lecture" discursive genre. Memorization processes are analyzed through discursive strategies. First, I focus on the "e-lecture" genre as a discursive genre based on memory and I then introduce the corpus and a few descriptive categories that correspond to discursive strategies for memorization. Second, I describe examples of discursive strategies that facilitate the efficient processing of new knowledge into existing cognitive structures and I consider the specificities of the web (dual-coding and usability) as a way to promote deep processing.
\end{abstract}

Keywords : knowledge transmission, learning theories, discourse analysis, e-lecture, memory

Mots clés : transmission des connaissances, théories de l'apprentissage, analyse du discours, e-conférence, mémoire

L'objectif de cet article est d'aborder la question de la mémoire à partir d'un genre discursif, l'e-conférence, appréhendé sous l'angle des théories de l'apprentissage et d'observer les stratégies discursives qui participent à la mise en œuvre des processus de mémorisation. Après avoir introduit l'e-conférence comme un genre mémoriel, on présentera le corpus ainsi que quelques catégories descriptives qui permettent de repérer ces stratégies. Ainsi, à partir d'exemples, on illustrera la manière dont le discours vise l'intégration de nouvelles connaissances au sein de réseaux cognitifs existants, s'appuie sur la construction structurelle des connaissances et prend en compte les spécificités du support web comme stimuli mémoriels.

\section{L'e-conférence : un genre mémoriel}

On commencera par présenter l'objet d'étude, l'e-conférence, puis on exposera brièvement les théories de l'apprentissage afin de situer cet 
objet par rapport à ces théories. On reviendra sur la place que le cognitivisme accorde à la mémoire et on mettra en regard mémoire et e-conférence. Le schéma suivant représente la démarche retenue : la mémoire, au centre de la discussion, est observée à partir d'un genre didactique à visée mémorielle, l'e-conférence, qui s'appuie lui-même sur une des trois théories de l'apprentissage, le cognitivisme, qui lui-même met au centre du traitement de l'information la mémoire.

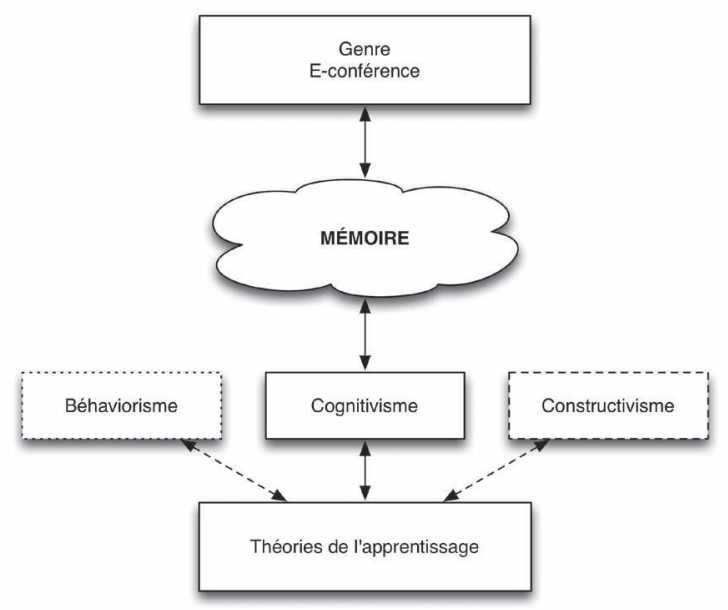

\subsection{L'e-conférence comme objet d'étude}

Parmi l'ensemble des discours de transmission des connaissances existants (Beacco et Moirand 1995), on restreint l'analyse aux discours didactiques (Moirand 1992) et à un genre particulier, l'e-conférence (de l'anglais «e-lecture »). Par définition, une conférence est un discours didactique qui expose et développe des connaissances dans un domaine particulier et s'adresse au grand public ou à un public spécialisé. Pour sa part, l'e-conférence constitue un prolongement sur le web de la conférence traditionnelle dans la mesure où on admet que les genres digitaux présentent une certaine stabilité par rapport à leurs pendants traditionnels écrits ou oraux (Yates et Sumner 1997, Rakotonoelina 2002).

Les travaux sur les conférences (traditionnelles) sont nombreux (voir par exemple Farrah 1990, Bligh 2000, Brown et Race 2002, Horgan 2003) et, sur le web, l'e-conférence est la forme d'enseignement la plus répandue parce qu'il s'agit d'une forme de formation et/ou d'autoformation rapide à mettre en œuvre et peu onéreuse (Driscoll et Carliner 2005, Carliner 2005). Dans les faits, elle se matérialise principalement par des productions discursives qui se répartissent en une succession ordonnée de pages web correspondant à un découpage des connaissances par paquets (Miller 1956); ces 
productions discursives sont dominées par l'écrit et sont donc destinées à la lecture (Goodfellow et Lea 2007).

Une e-conférence ne constitue pas nécessairement un site web et peut n'en constituer qu'une sous-partie - les autres parties pouvant être consacrées à d'autres e-conférences ou d'autres genres discursifs. De même, elle peut apparaître sur différents genres de sites dès lors qu'elle n'émane pas d'institutions reconnues historiquement et socialement comme formatrices. Ainsi, on les rencontre aussi bien sur des sites de services que sur des sites d'information, sur des sites personnels ou sur des sites communautaires - selon la typologie des genres de sites web proposée par Badre (2002) et Lawrence et Tavakol (2007) ${ }^{1}$. Il en découle qu'une e-conférence (qui se désigne rarement comme telle) est d'abord perçue comme un ensemble d'informations avant d'être considérée comme un ensemble de connaissances que l'on peut ou non acquérir. Pour reprendre les termes de Bligh (2000:20), on peut dire qu'une conférence sert avant tout à « enseigner de l'information » (de l'anglais « teach information »).

Cet exposé didactique tenu par un locuteur détenteur d'un savoir peut se définir comme un moyen pour un public d'acquérir une représentation de ce savoir. La mise en œuvre de cette représentation peut s'effectuer au travers de stratégies discursives qui favorisent les traitements mémoriels comme on le verra : il peut s'agir de l'organisation thématique de la conférence, de la récurrence de résumés, de cartes conceptuelles montrant l'articulation des concepts entre eux, etc. Mais cette mise en œuvre peut également prendre en compte différents styles cognitifs pour s'adapter aux différentes manières d'apprendre du public : cela peut concerner la présence du non-verbal, qui répond à un traitement mémoriel différent du verbal, mais également les principes qui sous-tendent l'utilisabilité du produit, s'il s'agit d'une e-conférence, etc.

Pour analyser le genre e-conférence, la démarche est la suivante : on ne part pas des configurations linguistiques et discursives comme cela se fait habituellement en linguistique du discours (Moirand 1990, 1994) pour aboutir à des interprétations (locales ou globales), mais de cadres interprétatifs empruntés au domaine des sciences de l'éducation. Ces cadres interprétatifs sont considérés comme des catégories interprétatives et servent aussi bien à construire des corpus qu'à orienter le regard de l'analyste et à penser la complexité des configurations linguistiques et discursives du genre considéré (voir par exemple Rakotonoelina 2008, 2009a, 2009b). Dans un deuxième temps, les configurations sont analysées à partir de catégories descriptives et les résultats de l'analyse permettent en retour de renseigner à la fois le genre considéré, les catégories interprétatives ayant servi de point de départ et la validité des catégories descriptives retenues.

1. Ce qui conduit à opérer sur le web une distinction entre genres de discours et genres de sites web. 
Lors de recherches précédentes, il s'agissait de rendre compte de la typicité et de la plasticité du genre e-conférence en considérant les différentes philosophies de l'éducation des adultes (voir par exemple Beder 1989, Zinn 1990, Spurgeon et Moore 1997, Conti 2007, Elias et Merriam 1980/2005) comme des catégories interprétatives corrélées à des configurations linguistiques et discursives, lesquelles permettaient de comprendre à la fois le genre et les philosophies. Pour observer le genre, ce sont ici les théories de l'apprentissage qui servent de point de départ, et en particulier les stratégies qui leur sont associées et qui favorisent les processus de mémorisation. En d'autres termes, les théories, tout comme les philosophies de l'éducation, servent de filtres pour analyser les textes.

\subsection{Théories de l'apprentissage}

Les théories de l'apprentissage sur lesquelles se basent les chercheurs en e-learning (voir par exemple Downing et Holtz 2008, Carliner 2008) fournissent une explication générale de la manière dont les apprenants réagissent à une présentation spécifique de contenus nouveaux dans des situations d'apprentissage particulières et offrent des moyens d'évaluer les résultats de l'apprentissage. Ces modèles sont rattachés à des écoles et chaque école élabore des cadres structurants (permettant à l'homme d'être plus performant) qui vont guider la conception de matériels pédagogiques. Aujourd'hui, on admet qu'en matière de modèles d'enseignement et d'apprentissage, trois écoles de pensée prédominent (Ertmer et Newby 1993, Ally 2008) :

- l'école de pensée béhavioriste influencée par Thorndike (1913), Pavlov (1927) et Skinner (1974) postule que l'apprentissage se traduit par des comportements observables ;

- en réaction à l'école béhavioriste, l'école de pensée cognitiviste postule que tous les résultats de l'apprentissage ne sont pas nécessairement observables, que l'apprentissage ne se réduit pas à un changement de comportement et qu'il résulte d'un processus qui implique, entre autres, pour traiter l'information des capacités mémorielles;

- l'école de pensée constructiviste (Doolittle 1999), que l'on considère soit du point de vue européen comme une école à part entière avec les conceptions piagétiennes, soit du point de vue nord-américain comme un prolongement du cognitivisme, postule que l'apprentissage est le résultat d'une interaction entre le sujet et son environnement, que l'apprenant construit son propre savoir et sa propre réalité en fonction d'expériences individuelles et sociales.

\subsection{E-conférences et cognitivisme}

Parmi ces trois théories de l'apprentissage et compte tenu de sa définition et de son mode de transmission (écriture/lecture), l'e-conférence intègre 
un modèle d'apprentissage de type cognitiviste. En effet, une e-conférence prend place dans ce que Clark $(2000,2002)$ appelle une architecture réceptive. L'architecture réceptive suppose que la formation consiste à fournir de l'information et que l'apprentissage consiste à « traiter » cette information. L'e-conférence suppose que le formateur est maître du savoir et que les apprenants acquièrent les connaissances qui leur sont transmises. C'est dans ce contexte particulier que la mémoire va jouer un rôle déterminant, l'e-conférence étant intrinsèquement un genre mémoriel, au sens cognitif du terme.

\subsection{Cognitivisme et mémoire}

Le cognitivisme (voir par exemple Jordan, Carlile et Stack 2008, Pritchard 2008, Weinstein et Acee 2008), issu de la psychologie cognitive (voir par exemple Braisby et Gellatly (eds) 2005), envisage l'apprentissage du point de vue du traitement de l'information et postule que le traitement de l'information s'opère par différents types de mémoire. Autrement dit, l'apprentissage, pour les cognitivistes, est un processus qui conduit à l'intégration d'informations nouvelles en mémoire (Sprenger 1999). Du modèle centré sur le traitement de l'information, on glisse par la suite vers un cognitivisme pédagogique représenté, entre autres, par Miller (1956), Ausubel (1960, Ausubel, Novak et Hanesian 1978) et Anderson (2000).

On revient très brièvement sur ce modèle fondé sur le traitement de l'information par les différents types de mémoire, car ce modèle permet, d'une part, de saisir la portée des stratégies d'apprentissage destinées à optimiser les processus de mémorisation et, d'autre part, d'observer les empreintes discursives de ces stratégies dans le genre e-conférence. Autrement dit, ces stratégies sont ici à considérer comme des catégories interprétatives générales qui permettent d'orienter le regard du chercheur lors de l'analyse du genre considéré, puisque ces catégories n'émanent pas de l'analyse elle-même mais existent en dehors d'elle et offrent un moyen de filtrer, d'observer et de comprendre autrement les discours de transmission des connaissances.

\subsection{Mémoire et e-conférences}

Le modèle de la mémoire le plus répandu est celui d'Atkinson et Shiffrin (1968).

Dans ce modèle, les stimuli sont d'abord traités par les registres sensoriels (la « mémoire sensorielle » - 1/4 à 1/2 seconde) qui filtrent l'information. Les stimuli encodés au niveau des registres sensoriels aboutissent dans ce que l'on appelle aujourd'hui la " mémoire de travail » (Dudai 2002, Hitch 2005) - Atkinson et Shiffrin parlaient, eux, de mémoire à court terme (0 à 18 secondes avec une capacité maximale de traitement de 7 éléments plus ou moins deux selon les travaux de Miller). Enfin, il 
faut que l'information soit traitée correctement au niveau de la mémoire de travail pour qu'elle soit ensuite transférée dans la « mémoire à long terme » (Kintsch, Patel et Ericsson 1999, Dudai 2002, Ruther 2005) l'aboutissement de toute information qui perdure au-delà d'une dizaine de secondes.
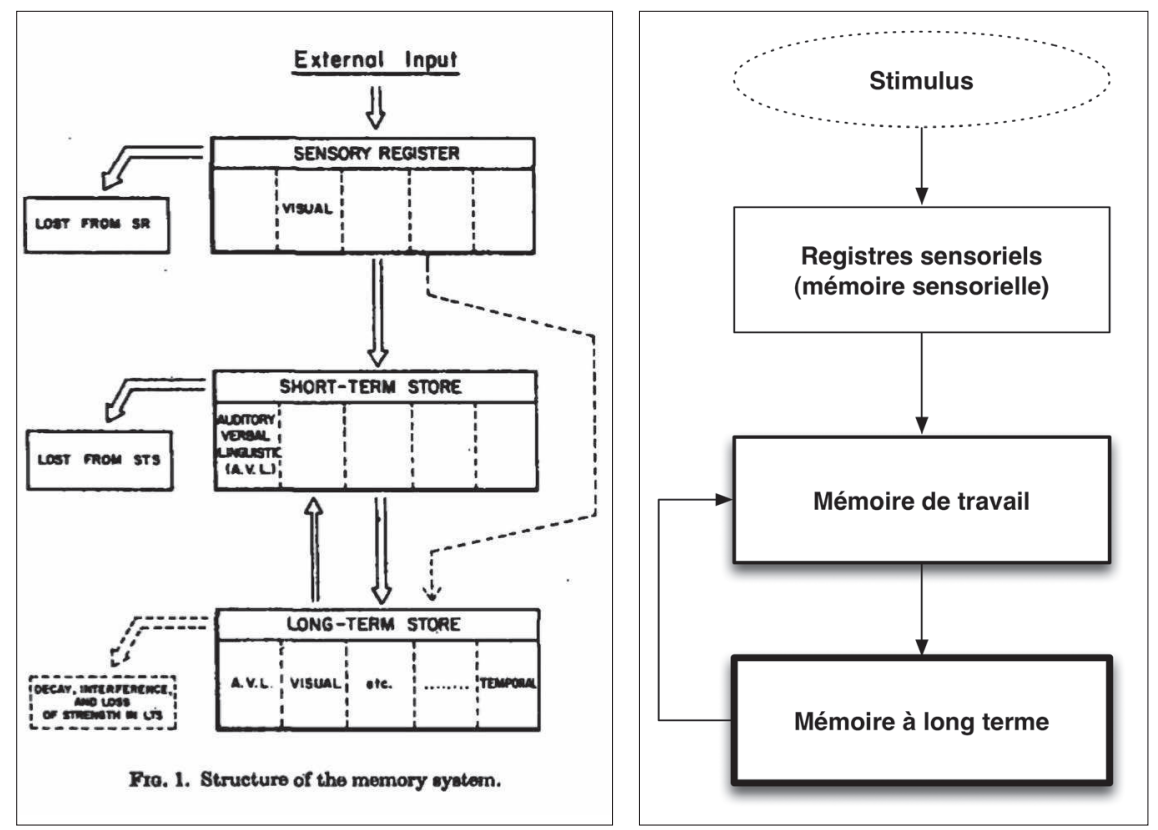

Lorsqu' on travaille sur les discours didactiques en e-learning et qu'on s'intéresse à la mémoire, l'objectif est donc d'observer, dans les textes, les stratégies discursives qui servent de stimuli aux apprenants de sorte que ces stimuli soient transférés depuis la mémoire sensorielle dans la mémoire de travail puis dans la mémoire à long terme. Mais ce n'est pas tout. Si l'information transférée dans la mémoire de travail dépend naturellement de l'attention que l'apprenant accorde aux discours, son transfert dépend aussi grandement des stratégies discursives mises en place pour respecter les structures cognitives d'un apprenant. Autrement dit, les discours didactiques sont supposés mettre en place conjointement deux types de stratégies : des stratégies servant de stimuli et des stratégies permettant l'intégration des connaissances dans la mémoire à long terme et notamment, dans le cadre des e-conférences, dans la mémoire déclarative (Dudai 2002) qui prend en charge la mémorisation des concepts. Si ces stratégies discursives de deux ordres, supports observables destinés à un traitement « adéquat » des connaissances par les différents types de mémoire, ne sont pas présentes, il y a peu de chance que l'apprentissage réussisse. 


\subsection{Choix d'un échantillon}

Les e-conférences s'appuient donc sur une approche directe des connaissances et non sur une approche basée sur la découverte active. Pour illustrer quelques stratégies discursives présentes dans les discours comme supports des processus de mémorisation, on a constitué un corpus autour de cinq e-conférences ${ }^{2}$. Ces cinq e-conférences relèvent d'une philosophie de l'éducation libérale et constituent des exemplaires typiques du genre e-conférence (Swales 1990, Rakotonoelina 2009b).

L'enseignement libéral se rencontre généralement dans le monde occidental au niveau académique, à l'université par exemple sous la forme de conférences, de lectures, de discussions, etc. Il s'agit, dans ce courant, de privilégier davantage la formation de l'esprit des apprenants plutôt que de les préparer à de véritables métiers ou à des carrières spécifiques. Les enseignements de ce type permettent, d'une part, de développer les connaissances fondamentales des apprenants sur le monde qui les entoure, d'autre part, de leur donner les moyens d'analyser et de comprendre le monde. L'enseignant est ici considéré comme l'expert, le détenteur d'un savoir.

- La première e-conférence « Le cerveau à tous les niveaux » est produite par l'Institut des neurosciences, de la santé mentale et des toxicomanies ainsi que par l'Institut de recherche en santé du Canada; on s'est intéressé à la section consacrée à la " Mémoire et apprentissage ».

- La seconde s'intitule «Les sciences de la vie au lycée » et est produite par le CNRS; on a observé la section consacrée aux « Régulations physiologiques $»$.

- La troisième intitulée « Webergos » porte sur l'ergonomie des interfaces web et est produite par Télécom et Management Sud Paris (ex Institut national des Télécom); on s'est centré sur la section consacrée à « L'écriture Web ».

- La quatrième intitulée « L'aventure des écritures » est produite par la Bibliothèque nationale de France; on a retenu la section « Matières et formes » et en particulier la rubrique « Supports et textes».

- Enfin, la cinquième intitulée "Comment écrire un rapport en français? » est produite par des enseignants appartenant à l'European University Institute (Italie), à l'University College London (Angleterre) et à l'université d'Oviedo (Espagne); c'est le module 5 « Comment situer vos idées et celles des autres? » qu'on a examiné.

\subsection{Choix des observables comme reflets des structures cognitives}

Dans le cadre d'un apprentissage qui s'appuie exclusivement sur une e-conférence, celle-ci oblige à considérer le discours comme seul

2. Les références sont données en fin d'article. 
médiateur des connaissances. Par conséquent, le discours doit créer les conditions d'apprentissage favorables par la mise en œuvre de stratégies qui orientent l'activité de l'apprenant. Généralement, cela se traduit par des stratégies discursives qui s'appuient, entre autres, sur des variables « mathémagéniques », c'est-à-dire des éléments qui permettent d'assimiler les connaissances au rang desquels on peut citer, par exemple, les structurants antérieurs (objectifs, questions posées avant l'apprentissage, rappel des prérequis, etc.) et les structurants postérieurs (synthèses, généralisation, élargissement, etc.).

Certaines des stratégies discursives relevées passent aujourd'hui pour des évidences, mais c'est parce qu'elles s'inscrivent, entre autres, dans un genre dont les représentations sont largement partagées qu'elles sont justement évidentes (au sens où le système éducatif nous a exposés à ce genre). Cela dit, dans les années 1960, ces stratégies apparaissaient comme originales et révolutionnaires par rapport aux approches préconisées par le béhaviorisme. Ce sont donc les traces de ces évidences qu'on cherche à repérer dans les discours et qui sont autant d'entrées descriptives à questionner dans une perspective d'analyse des discours didactiques.

Trois types de stratégies permettront d'illustrer la relation entre le genre e-conférence et les processus de mémorisation :

- les stratégies qui consistent à permettre l'intégration de nouvelles connaissances dans des réseaux de connaissances existants;

- les stratégies qui consistent à mettre en place une construction structurelle des connaissances;

- et enfin, les stratégies qui exploitent les potentialités sémiotiques du support web pour agir comme stimuli d'apprentissage pour un traitement mémoriel.

\section{Intégration de nouvelles connaissances dans des réseaux existants}

Les recherches cognitives ont montré que l'organisation des connaissances au sein de la mémoire s'opère sous la forme de réseaux et que l'apprentissage de nouvelles connaissances est dépendant des connaissances antérieures. Ainsi, l'acquisition de nouvelles connaissances passe par une mobilisation de connaissances antérieures relatives au même domaine et ces nouvelles connaissances intègrent les réseaux existants et/ou les réorganisent pour prendre en compte les contraintes des nouvelles connaissances (Bruner 1960, Ausubel et al. 1978).

Au niveau discursif, on peut observer quatre phénomènes : des stratégies qui vont du connu à l'inconnu, des stratégies qui vont du simple au complexe, des stratégies qui vont du général au particulier ou des stratégies qui vont du concret au particulier. Ces quatre stratégies ne sont pas exclusives l'une de l'autre et peuvent se recouper. On s'appuiera ici sur l'econférence consacrée au cerveau pour illustrer ces cas de figure en discours. 


\subsection{Du connu à l'ínconnu}

Exemple 1. Le cerveau à tous les niveaux

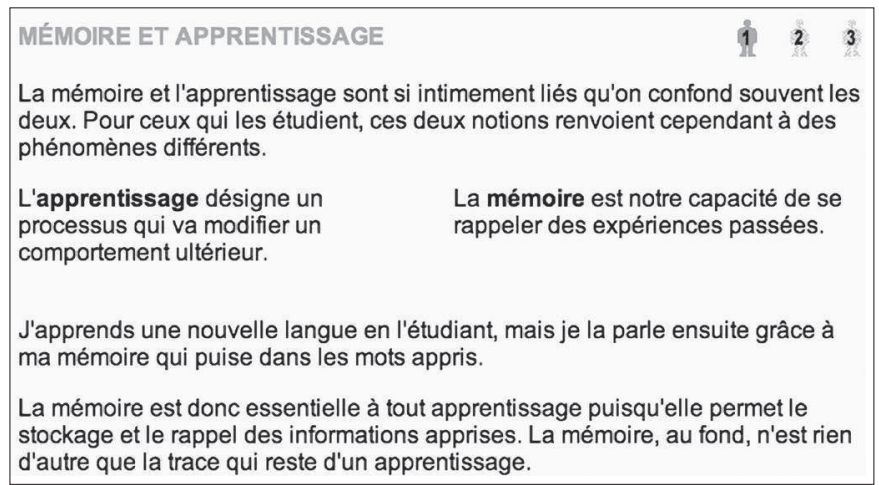

Dans cet extrait, qui marque le début d'une section consacrée à « la mémoire et l'apprentissage » destinée aux débutants, on observe la manière dont le discours opère un pont entre des représentations censées être partagées par des néophytes « on confond souvent les deux, mémoire et apprentissage » et la réalité telle qu'elle est conçue par les spécialistes « ces deux notions renvoient cependant à des phénomènes différents ». Cette distinction apparaît de façon nette par la mise en page web puisque les définitions de la mémoire et de l'apprentissage ne se font plus de manière linéaire dans l'aire de la page mais en deux blocs côte à côte. La distinction n'est donc pas seulement conceptuelle, elle est également visuelle, avec deux stimuli visuels supplémentaires qui se superposent à travers la graisse des termes « apprentissage » et « mémoire ».

Le connu se manifeste également à travers l'énoncé d'exemplification « J'apprends une nouvelle langue en l'étudiant, mais je la parle ensuite grâce à ma mémoire qui puise dans les mots appris » en mettant l'apprenant dans une situation de vécu et en illustrant les définitions proposées.

\subsection{Du simple au complexe}

Exemple 2. Le cerveau à tous les niveaux (débutant : couleur orange)

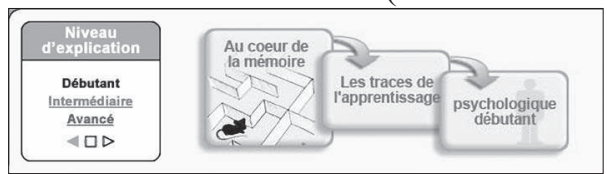

Exemple 3. Le cerveau à tous les niveaux (intermédiaire : couleur verte)

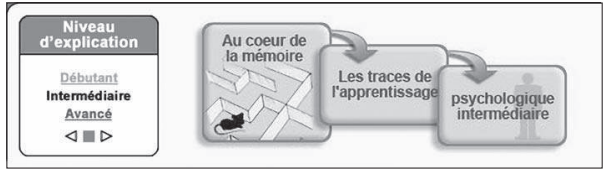


Exemple 4. Le cerveau à tous les niveaux (avancé : couleur rouge)

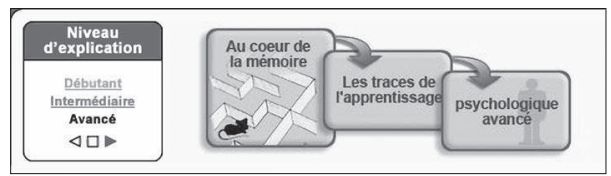

Cette e-conférence - ce n'est pas le cas de toutes les e-conférences retenues dans l'échantillon - s'appuie sur des niveaux de connaissances. Ainsi l'interface montre que l'e-conférence possède trois « niveaux d'explication » identifiés en outre par une couleur spécifique : orange pour un niveau d'explication débutant, vert pour un niveau d'explication intermédiaire et rouge pour un niveau d'explication avancé. Dans tous les cas, cette e-conférence prend en compte les différents styles cognitifs de l'apprenant, même s'il est possible pour tout apprenant de suivre la progression du simple au complexe proposée.

Exemple 5. Le cerveau à tous les niveaux (débutant)

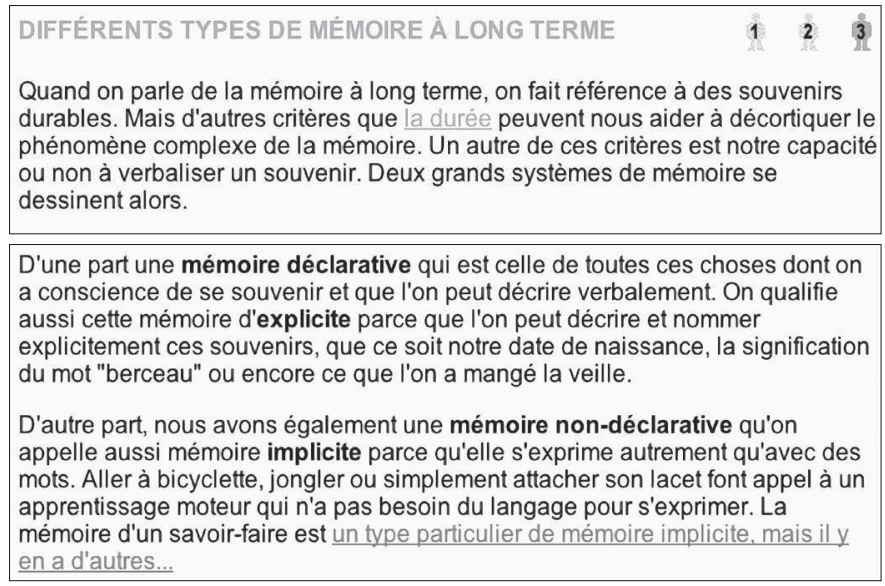

Lorsqu'on observe les niveaux d'explication, on constate que l'on glisse progressivement du simple au complexe, comme on le voit dans cet exemple consacré aux différents types de mémoire à long terme par subdivision : la mémoire à long terme se dessine en deux nouveaux paragraphes parfaitement identifiables visuellement et introduit linguistiquement par « d'une part » et par « d'autre part »; quant à l'identification des types de mémoire, elle se fait également visuellement par la graisse puisque se détachent du reste les termes « mémoire déclarative »/ " explicite » dans le premier paragraphe et « mémoire non-déclarative » / « implicite » dans le second.

Ce voyage cognitif du simple au complexe se matérialise aussi par la présence de l'hypertextualité qui reprend le code des couleurs précédemment mentionné; ainsi l'apprenant a la possibilité de cliquer sur le prédicat 
« un type particulier de mémoire implicite, mais il y en a d'autres... » pour obtenir un niveau d'explication plus avancé. On passe donc de l'orange/ débutant au vert/intermédiaire.

Ce passage amène à rendre compte du général au particulier, qui est une stratégie qui se superpose plus souvent qu'elle ne se distingue de celle qui va du simple au complexe.

\subsection{Du général au particulier}

Cette stratégie rejoint le principe de la différentiation progressive chez Ausubel (Ausubel et al. 1978 : 190) : les idées générales sont d'abord présentées puis des différences sont établies au fur et à mesure. Pour Ausubel, l'efficacité d'une telle approche tient essentiellement au fait que la structure cognitive est elle-même organisée selon un principe hiérarchique basé sur la différenciation progressive. Ainsi, dans la continuité de l'exemple précédent, lorsqu'on clique sur le prédicat d'invitation à aller de l'avant, on constate que chaque niveau de connaissance forme un tout autonome, puisqu'on retrouve exposées les mêmes différences entre les types de mémoire.

\section{Exemple 6. Le cerveau à tous les niveaux (intermédiaire)}

L'une de ces distinctions qui apparaissent comme des plus fondamentales est celle que
I'on fait entre les mémoires explicites (ou déclaratives) et celles qui sont implicites (ou
non-déclaratives), selon que l'on peut ou non en exprimer verbalement le contenu.
Traditionnellement, les études se sont concentrées sur la mémoire explicite où nous
pouvons nous rappeler consciemment les faits et les choses. On demande par exemple à
quelqu'un de mémoriser un matériel donné (liste de mots, images, etc.) qu'il doit ensuite
se remémorer verbalement. Cette mémoire semble donc favoriser l'encodage
d'informations relatives à l'identité, la fonction et les attributs d'un objet.
Plus récemment, un intérêt croissant en recherche a vu le jour pour une forme
inconsciente de mémoire appelée mémoire implicite. Il s'agit d'une forme de mémoire où
l'on ne retient pas l'expérience qui en est à l'origine. De plus, le rappel d'un souvenir
encodé dans la mémoire implicite se fait automatiquement, sans les efforts de rappel
nécessaire à la mémoire explicite.

La mémoire procédurale, qui permet l'acquisition d'habiletés et l'amélioration
progressive de ses performances motrices, est peut-être la mieux connue des différents
types de mémoires implicites. C'est cette mémoire qui permet, par exemple, de conduire
sa voiture ou de manger sans devoir être totalement concentré sur ces tâches. La
mémoire procédurale est inconsciente, non pas au sens freudien de souvenir refoule,
mais parce qu'elle est constituee d'automatismes sensorimoteurs si bien intégrés que
nous n'en avons plus conscience. La mémoire procédurale est souvent préservée chez
les patients souffrant d'amnésie profonde, ce qui plaide pour un système de voies
nerveuses distinct.
Beaucoup de nos conditionnements émotionnels et de nos réflexes conditionnés
sont également du domaine de la mémoire implicite. L'apprentissage associatif qui est à
la base de ces formes de mémoire est un processus très ancien phylogénétiquement qui
peut se faire sans l'intervention de la conscience.
L' acquisition d'un souvenir dans la mémoire implicite se fait à notre insu et elle doit
souvent être mise en évidence de manière indirecte, par des phénomènes comme
l'amorçage ("priming"). L'effet d'amorçage est une augmentation de la précision ou de la
vitesse d'une prise de décision qui survient suite à l'exposition préalable d'une information
pertinente sur le contexte, mais sans qu'il n'y ait aucune motivation à rechercher cette
information de la part du sujet. Par exemple, la décision que la chaîne de caractères
"docteur" est un mot est prise plus rapidement lorsque la chaîne de caractères qui le
précede etait "infirmière" comparé a "nord" ou à une chaîne de caractères quelconque
comme "nuber".
La mémoire explicite est quant à elle souvent subdivisée en mémoire épisodique et
sémantique...


Le premier paragraphe de l'exemple introduit les différences, les deux paragraphes suivants les développent avec la même logique visuelle que précédemment. Ces trois premiers paragraphes qui présentent les deux grandes divisions de la mémoire à long terme sont à ranger, dans ce contexte, au rang du général déjà présent dans le niveau d'explication débutant. On bascule précisément dans le particulier à partir du quatrième paragraphe où les termes « mémoire procédurale »/《 effet d'amorçage » et « conditionnements émotionnels »/《 réflexes conditionnés » vont constituer de nouvelles subdivisions conceptuelles.

On constate qu'au niveau intermédiaire seule la mémoire implicite fait l'objet d'un développement (et non la mémoire explicite). C'est au niveau avancé que sera développée la mémoire explicite comme l'annonce le dernier énoncé dont une partie apparaît en hypertexte de couleur rouge : « La mémoire explicite est quant à elle souvent subdivisée en mémoire épisodique et sémantique... ».

La question de savoir pourquoi la mémoire explicite fait l'objet d'un traitement dans un niveau d'explication avancé est due, selon moi, à deux raisons : la première tient au fait que l'on évite de multiplier l'information au niveau intermédiaire pour éviter une surcharge cognitive; la seconde, qu'on va maintenant développer, tient au fait qu'il y a, dans un apprentissage, un passage du concret à l'abstrait, le concret étant un mode de représentation plus accessible que l'abstraction.

\subsection{Du concret à l'abstrait}

Dans ce niveau avancé d'explication consacrée aux différents types de mémoire, les premières subdivisions sont reprises mais laissent place cette fois à un développement de la mémoire explicite. Alors que dans le niveau intermédiaire, on avait des énoncés qui s'accrochaient au réel, comme par exemple, «C'est cette mémoire [procédurale] qui permet, par exemple, de conduire sa voiture ou de manger sans devoir être totalement concentré sur ces tâches », l'essentiel du propos dans le développement de la mémoire explicite tend vers un mode de représentation plus abstrait ou symbolique, pour reprendre Bruner (1960), où le langage endosse pleinement un rôle de représentant symbolique de la connaissance. C'est ainsi que l'on va lire par exemple concernant la mémoire sémantique, forme de mémoire explicite (avant-dernier paragraphe de l'exemple 7) :

C'est aussi la mémoire des règles et des concepts qui permet la construction d'une représentation mentale du monde sans la perception immédiate. Ce contenu est donc abstrait et relationnel, et il est associé à la signification des symboles verbaux. 
Exemple 7. Le cerveau à tous les niveaux (avancé)

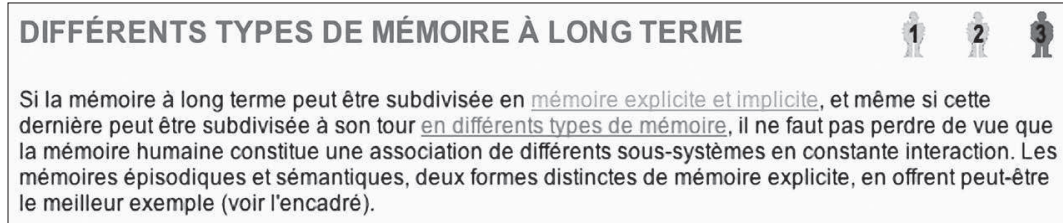

La mémoire épisodique, parfois appelẻe autobiographique, permet à un sujet de se rappeler des événements qu'il a personnellement vécus dans un lieu et à un instant donné. C'est le souvenir de ce qu'on a mangé la veille, le nom d'un ancien camarade de classe ou encore la date d'un événement public marquant.

La caractéristique la plus distinctive de la mémoire épisodique est que lindividu se voit en tant qu'acteur des événements mémorisés. Par conséquent, le sujet mémorise non seulement un evénement qu'il a vécu, mais tout le contexte particulier de cet événement.

C'est cette composante de la mémoire qui est le plus souvent touchée par les amnésies. De plus, la charge émotionnelle vécue par le sujet au moment des faits conditionne la qualité de la mémorisation épisodique.

La mémoire sémantique est le système par lequel l'individu stocke sa connaissance du monde. C'est
une base de connaissances que nous possédons tous et dont une grande partie nous est accessible
rapidement et sans effort. C'est la mémoire du sens des mots, celle qui nous permet de se souvenir du
nom des grandes capitales, mais aussi des coutumes sociales, de la fonction des choses, de leur couleur
ou de leur odeur.
C'est aussi la mémoire des règles et des concepts qui permet la construction d'une représentation
mentale du monde sans la perception immédiate. Ce contenu est donc abstrait et relationnel, et il est
associé à la signification des symboles verbaux.
La mémoire sémantique est indépendante du contexte spatio-temporel de son acquisition. Comme il
s'agit d'une mémoire de référence qui renferme des informations accumulées de façon répée durant
toute notre vie, la mémoire sémantique est habituellement épargnée par les amnésies. Mais certaines
démences peuvent l'affecter (voir l'encadré).

Les quatre stratégies que l'on vient d'exposer montrent que l'intégration de nouvelles connaissances dans des réseaux de connaissances existants se fait de deux manières dans les discours : soit par connexion à des réalités expérientielles ou à des représentations mentales, soit par connexion aux réseaux de connaissances développés à l'intérieur de l'e-conférence ellemême par le passage d'un mode (connu, simple, général et concret) à un autre (inconnu, complexe, particulier et abstrait).

\section{Construction structurelle des connaissances}

Les cognitivistes accordent une importance particulière à la notion de structure. Pour que les connaissances perdurent au niveau de la mémoire à long terme, l'information doit être structurée et les stratégies discursives doivent permettre à l'apprenant de saisir la structure des connaissances qu'il aura à assimiler en mettant en évidence non seulement les concepts essentiels mais également en établissant des liens entre ceux-ci. Dès lors, on peut s'intéresser par exemple à la macrostructure et à la mésostructure (Dumortier 2001) de l'e-conférence ou bien encore aux « structurants antérieurs et postérieurs $\gg$. 


\subsection{Macrostructure et mésostructure}

Une e-conférence est un ensemble de connaissances planifiées et sa macrostructure se caractérise par un parcours d'appropriation des savoirs. En tant qu'exposé didactique, cette macrostructure est déterminée par le paradigme explicatif et par les concepts cruciaux du domaine traité.

Exemple 8. Comment écrire un rapport? (macrostructure)

Sommaire général
Remarques préliminaires
allez-vous produire?
Module 2 : Comment présenter votre
rapport?
Module 3 : Comment situer vos idées
Module 4 : Comment structurer votre
rapport?
Module 5 : Comment faire pour qu'un
Moxte soit cohérent et dynamique?
Module 6 : La bibliographie
Annexes, illustrations et légendes,
index

La macrostructure de l'e-conférence «Comment écrire un rapport en français? » s'articule principalement autour d'une série de questions : « Comment présenter votre rapport? »; «Comment situer vos idées et celles des autres? », etc. Ces questions sont censées être celles que l'apprenant se pose. À ce titre, cette macrostructure joue un double rôle : un rôle d'interpellation (une question, fût-elle rhétorique, vise toujours une fonction d'appel) et un rôle de structuration des contenus. Néanmoins, cette macrostructure montre que les connaissances sont thématiquement indépendantes et ne suivent pas une progression particulière, permettant à l'apprenant d'entrer dans l'e-conférence selon ses propres questionnements : « Comment situer vos idées et celles des autres? » n'a pas nécessairement de lien logique avec «Comment faire pour qu'un texte soit cohérent et dynamique?».

Exemple 9. Webergos (macrostructure)

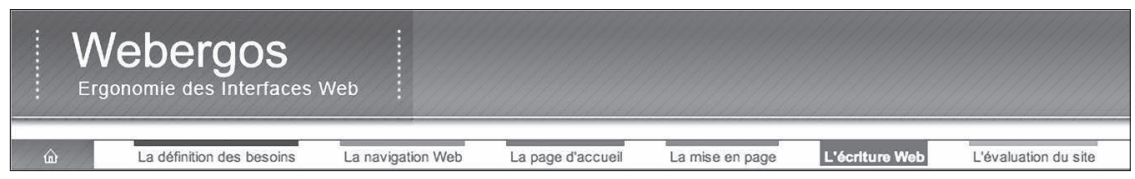


La macrostructure de Webergos, ergonomie des interfaces web, peut être considérée comme un modèle du genre en matière de structuration des connaissances : cinq titres; cinq syntagmes nominaux; cinq couleurs. De fait, la programmation des connaissances développées par l'e-conférence peut être aisément traitée en mémoire. Certes, il s'agit d'un site consacré à l'ergonomie, mais on voit là une mise en œuvre des travaux de Miller : la capacité de stockage est limitée à sept items plus ou moins deux dans la mémoire à court terme.

La mésostructure correspond souvent à des sections, des soussections, etc. Elle dépend de la macrostructure et les paquets d'informations qu'elle inclut ou qui l'incluent dans des rapports descendants ou ascendants rendent le niveau de la mésostructure complexe. Au-delà des stratégies discursives mises en œuvre, le traitement cognitif de la mésostructure est en partie déterminé par les capacités de l'apprenant de la rapporter à ce qui précède et à ce qui suit, de voir à quel niveau d'enchâssement elle se situe.

Exemple 10. Comment rédiger un rapport? (mésostructure du « Module $3 »$ )

Module 3 : Comment slituer vos
Ides ot celles des autres?
Lemarques préliminaires
Lestinataires
D'où viennent nos idées?
Dialogue avec vos prédécesseurs et
caractère polyphonique des textes
Les citations pour s'appuyer sur
une autorité et développer des idées
nouvelles
Comment positionner votre propre
discours?
Litations, commentes, leur relation aux
les mettre?

Exemple 11. Webergos (mésostructure de «L'écriture Web »)

\begin{tabular}{|c|c|c|c|c|c|c|}
\hline 内 & La définition des besoins & La navigation Web & La page d'accueil & La mise en page & L'écriture Wob & L'évaluation du site \\
\hline \multicolumn{2}{|c|}{ LECRTUREWEB } & \multicolumn{5}{|l|}{ PRINCIPES } \\
\hline \multicolumn{2}{|c|}{ 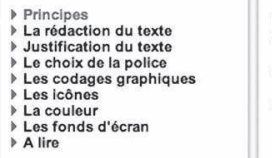 } & \multirow{3}{*}{\multicolumn{5}{|c|}{$\begin{array}{l}\text { L'écriture web est différente de l'écriture d'un article ou d'un livre. II n'existe cependant pas une seule écriture web, } \\
\text { cela serait certainement plus simple. } \\
\text { Cela signifie quill est difficile de généraliser en quelques règles ce que vous allez vouloir "écrire". } \\
\text { Selon J. Nielsen l'écriture Web engage trois grands principes : } \\
\quad \rightarrow \text { la concision, selon J. Nielsen et Morkes nous lisons } 25 \% \text { plus lentement à l'écran. } \\
\quad \rightarrow \text { la balayabilité, quand nous lisons, nous "scannons", nous lisons aussi en diagonale dit-on, } 79 \% \text { d'entre } \\
\text { nous balaient les textes. In' y a que } 16 \% \text { des "lecteurs Web" qui lisent mot à mot tout le contenu qui leur est } \\
\text { présenté. } \\
\quad \rightarrow \text { le morcellement des paqes. les internautes n'utilisent pas svstématiauement l'ascenseur vertical. cela }\end{array}$}} \\
\hline & n du site & & & & & \\
\hline & & & & & & \\
\hline
\end{tabular}

Si l'on compare les deux mésostructures, celle de «Comment écrire un rapport? » et celle de Webergos, celle de Webergos respecte la logique des sept items plus ou moins deux, liée à une lisibilité écran maximale, 
et présente un pouvoir de captation mémorielle plus important grâce à la brièveté des données linguistiques.

Dans une e-conférence, la macrostructure peut se combiner simultanément à la mésostructure permettant à l'apprenant de toujours se repérer par rapport à la structure d'ensemble des connaissances. C'est ce qui se produit dans l'e-conférence « L'aventure des écritures ».

Exemple 12. L'aventure des écritures (macrostructure et mésostructure intégrales)

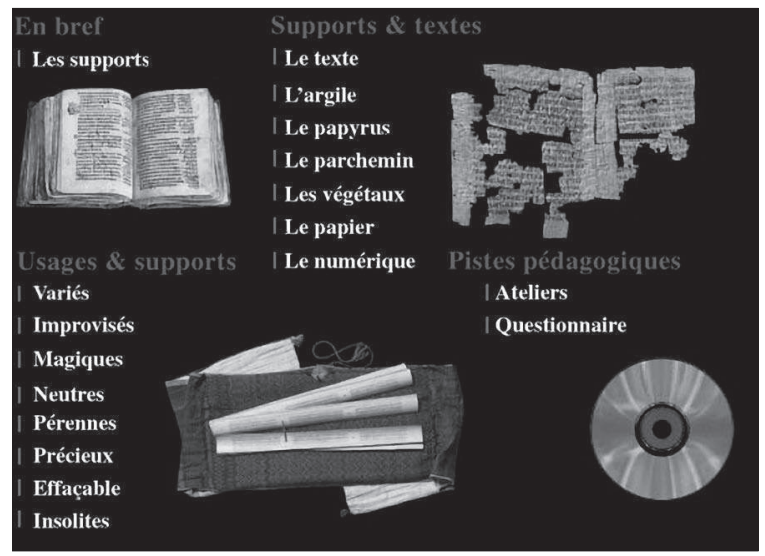

La page d'accueil montre une macrostructure en quatre entrées qui se détachent, par le jeu des couleurs, de la mésostructure dont les items des principales entrées « Supports et textes » et «Usages et supports » varient de sept à huit. La saisie mémorielle de ces items est renforcée par la cohérence des catégories linguistiques choisies : syntagmes nominaux dans un cas, adjectifs dans l'autre.

Exemple 13. L'aventure des écritures (macrostructure simplifiée et mésostructure)

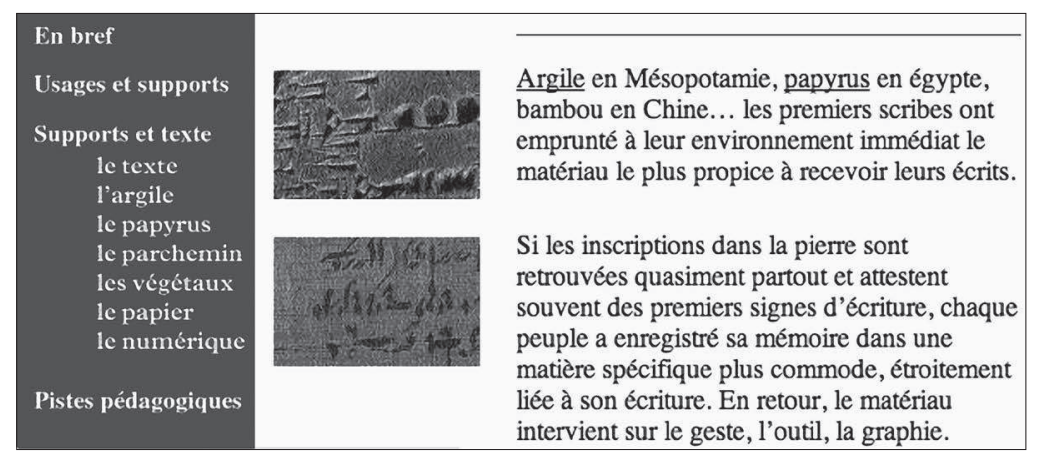

Lorsqu'on clique sur un item et qu'on entre dans le corps de l'e-conférence apparaît un menu de navigation; ne subsistent alors que les éléments 
de la macrostructure, et seuls les éléments de la mésostructure de l'item macrostructurel consulté demeurent, offrant un accès permanent aux types de supports.

\subsection{Structurants (antérieurs et postérieurs)}

Les structurants antérieurs pour Ausubel (1960) réfèrent à ces textes courts, schémas, graphiques, généralement présentés en début de chapitres, de modules, de sections, qui facilitent la mise en relation des éléments qui font l'objet de l'apprentissage et qui assurent le lien avec les éléments déjà maîtrisés disponibles dans la structure cognitive de l'individu. Les structurants postérieurs sont les contreparties qui se situent en fin de chapitres, sections, etc.

Exemple 14. Comment rédiger un rapport?

\begin{tabular}{|c|c|}
\hline$\mapsto$ & Module 3 : Remarques préliminaires \\
\hline Sommaire général & $\begin{array}{l}\text { Je soit le type de rapport à rédiger, le rédacteur doit être conscient du fait } \\
\text { cherche à établir une relation avec ses destinataires et à obtenir leur }\end{array}$ \\
\hline Remarques préliminaires & ésion, \\
\hline $\begin{array}{l}\text { Module 1: Quel type de rapport } \\
\text { allez-vous produire? }\end{array}$ & $\begin{array}{l}\text { qu'une bonne partie de son information vient des autres, } \\
\text { que, par conséquent, il lui faut maitriser la façon dont il garde les traces de }\end{array}$ \\
\hline $\begin{array}{l}\text { 1 Module } 2 \text { : Comment présenter votre } \\
\text { rapport? }\end{array}$ & $\begin{array}{l}\text { ses lectures, dès le départ (voir le module } 6 \text { pour établir les notices } \\
\text { bibliographiques). }\end{array}$ \\
\hline \multirow{3}{*}{$\begin{array}{l}\text { Module } 3 \text { : Comment síher vos } \\
\text { dées et celles des autres? } \\
\text { "zemarques préliminaires } \\
\text { La relation entre auteur et } \\
\text { destinataires } \\
\text { D'où viennent nos idées? } \\
\text { Dialogue avec vos prédécesseurs et } \\
\text { caractere polyphonique des textes } \\
\text { Les citations pour s'appuyer sur } \\
\text { une autorité et développer des idées } \\
\text { nouvelles } \\
\text { Comment positionner votre propre } \\
\text { discours? } \\
\text { Les notes, leur relation aux } \\
\text { citations, comment les rédiger et où } \\
\text { les mettre? }\end{array}$} & $\begin{array}{l}\text { Il lui faut relever dans le texte consulté des citations potentielles et les conserver } \\
\text { pour enrichir son texte ou pour des notes en bas de page. Cela demande une } \\
\text { discipline minutieuse. Dans tous les cas, le rédacteur deva suivre } \\
\text { rigoureusement, d'un bout à l'autre du document, le même procédé de } \\
\text { présentation des références. La collecte de citations apporte, à double titre, un } \\
\text { fort enrichissement du discours. D'une part, les passages relevés renforcent } \\
\text { l'auteur dans sa conviction, d'autre part, ils sont l'occasion de développer la } \\
\text { pensée dans des directions imprévues à partir du point de vue d'un autre auteur } \\
\text { mais pas toujours dans la même direction que lui. }\end{array}$ \\
\hline & $\begin{array}{l}\text { Par ailleurs, pour souligner la cohérence de son discours et produire une forte } \\
\text { cohésion du texte, le rédacteur s'appuiera sur des connecteurs (voir module 5). }\end{array}$ \\
\hline & $\begin{array}{l}\text { Enfin un travail de réécriture l'attend. C'est une phase impérative de la } \\
\text { rédaction (voir encore dans le module } 5 \text { le paragraphe sur la réécriture). }\end{array}$ \\
\hline
\end{tabular}

Dans l'échantillon considéré, seule l'e-conférence « Comment rédiger un rapport? » comporte des structurants antérieurs verbaux et aucun structurant postérieur n'a été rencontré. De ce point de vue, on peut se demander si les structurants postérieurs (verbaux) font partie du genre e-conférence. Dans cet exemple, le structurant antérieur est désigné par « Remarques préliminaires » et s'il annonce bien la structure des connaissances que l'on va trouver dans le module, il va au-delà, puisque la structuration des nouvelles connaissances se fait en lien avec d'autres connaissances présentes dans d'autres modules - qui ne sont d'ailleurs pas nécessairement des connaissances déjà vues.

Si les catégories (connexions cognitives et connexions structurelles) que l'on vient d'observer dans le genre e-conférence doivent être considérées comme des supports pour un traitement mémoriel des connaissances, l'inscription de l'e-conférence sur le web oblige à adopter une approche du discours comme un tout sémiotiquement hétérogène (Herring 2009). En effet, on peut appréhender ces catégories certes dans une perspective 
linguistique et textuelle, mais on peut également prendre en compte d'autres éléments dans les e-conférences qui vont permettre de structurer ces connaissances, à l'instar des tableaux, des schémas, des graphiques, des cartes conceptuelles, etc., qui servent à la fois de stimuli pour les registres sensoriels et qui permettent de représenter les connaissances autrement que par la linéarité de la chaîne verbale.

\section{Stimuli mémoriels et support web}

L'inscription d'une e-conférence sur le support web offre des possibilités graphiques que ne permet pas toujours l'imprimerie pour des raisons de coût. Le web se projette sur écran et les images constituent en ce sens des stimuli exploitables à des fins mémorielles.

\subsection{Pluriencodage des connaissances}

La question des graphiques dans l'analyse des discours de transmission des connaissances est encore peu présente. Quand les graphiques ont été traités, ils l'ont surtout été du point de vue de leur complémentarité par rapport au texte (voir par exemple Jacobi 1985, 1999, Petiot 1992, Rakotonoelina 1999). Dans tous les cas, le traitement était surtout linguistico-centré, au sens où l'appréhension des graphiques s'opérait à partir d'un ancrage linguistique et la question était de se demander ce que pouvaient bien apporter les graphiques par rapport au texte et non l'inverse.

Or, les cognitivistes spécialistes de l'éducation comme Paivio (1986) qui travaille sur le double encodage ou Clark et Mayer (2008) qui travaillent plus spécifiquement sur l'e-learning montrent que le double encodage permet non seulement de prendre en compte des styles cognitifs d'apprenants différents, mais aussi et surtout de faciliter le traitement de l'information par la mémoire de travail et son transfert dans la mémoire à long terme précisément parce que l'information doublement codée est traitée par des zones du cerveau différentes.

Il n'est pas rare de rencontrer différents types de graphiques dans les e-conférences, chaque type ayant une fonction cognitive précise - cas des graphiques représentatifs ou des graphiques organisationnels - ou pouvant cumuler plusieurs fonctions - cas des graphiques polyfonctionnels.

Dans l'exemple 15, la photo du manuscrit peut être considérée comme un graphique représentatif dont l'objectif est de « représenter » 1'apparence visuelle d'un élément unique. La connexion entre le texte et la photo s'opère par le titre et par le déictique « ce » (« ce manuscrit »). Un tel graphique rend visuellement accessible ce qui ne l'est pas et ne le sera sans doute jamais pour qui s'intéresse aux écritures et à leurs supports. 
Exemple 15. L'aventure des écritures (graphique représentatif)

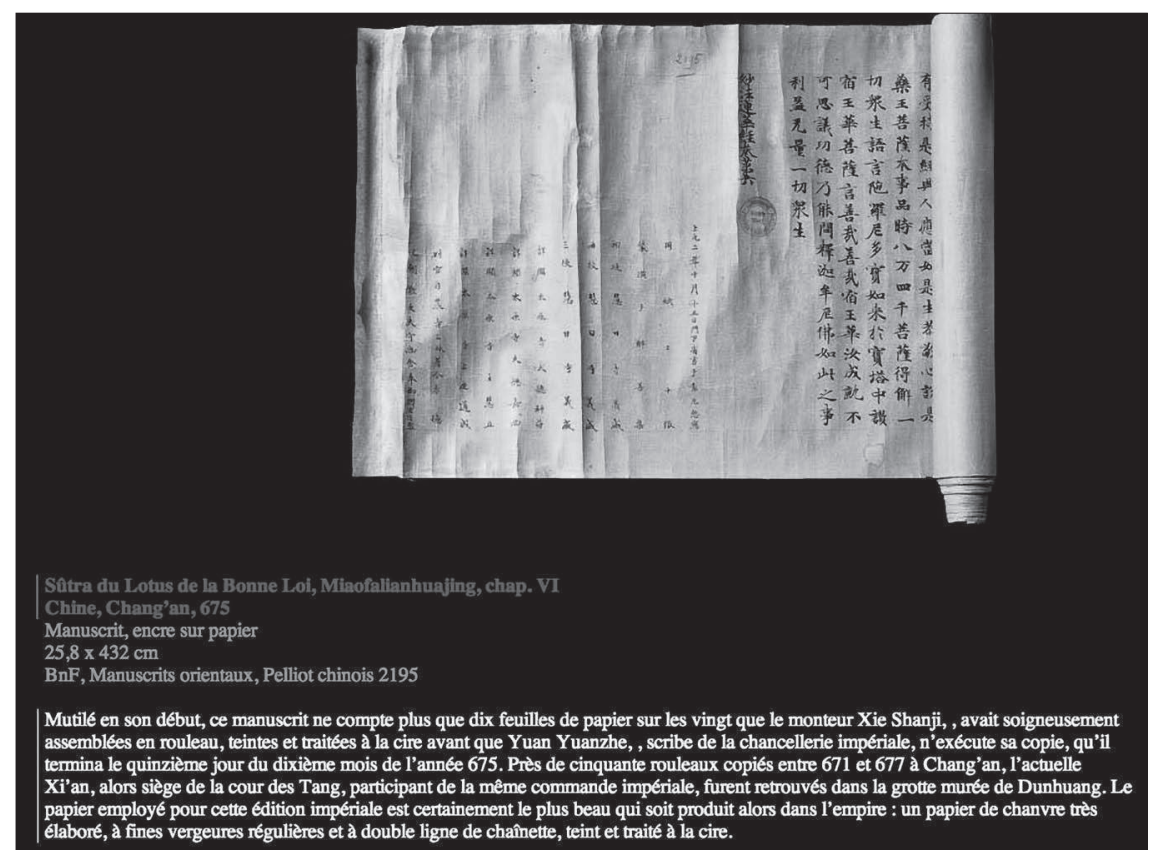

Exemple 16. Le cerveau à tous les niveaux (graphique organisationnel à partir d'un schéma)

Cette capacité de retenir temporairement une information en vu de mener à bien une tâche est spécifiquement humaine. Elle rend très active certaines régions de notre cerveau, en particulier le lobe préfrontal.

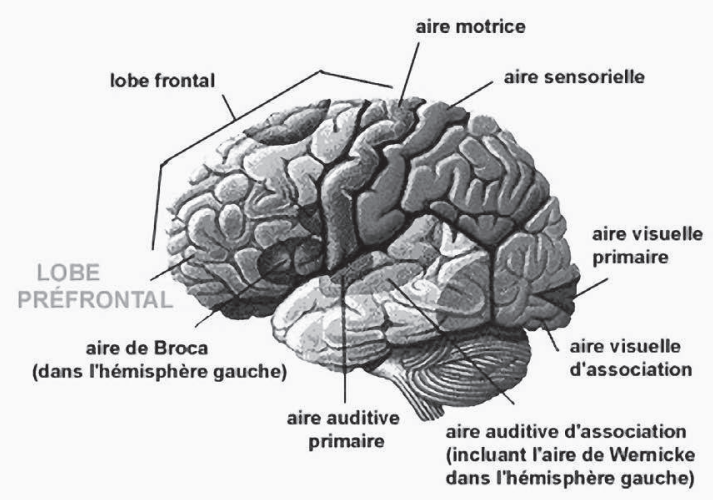

Cette région située tout en avant du cerveau est très développée chez l'être humain. C'est elle qui nous donne notre grand front droit plutôt que le front fuyant de nos cousins primates. II n'est donc pas étonnant que la région du cerveau qui semble la plus active dans une des activités les plus humaines soit située justement dans cette région préfrontale qui n'est bien développée que chez nous...

Mais la mémoire humaine est complexe et d'autres régions du cerveau sont évidemment impliquées... 
Plus complexes, les graphiques organisationnels montrent des relations qualitatives entre différents éléments. Il peut s'agir d'un schéma, comme c'est le cas pour le lobe préfrontal représenté dans l'exemple 16 : l'accent s'opère certes sur le lobe préfrontal, mais le schéma permet de repérer le lobe préfrontal dans sa relation au lobe frontal et aux différentes aires. Les aires (considérées comme autant de connaissances différentes) se structurent visuellement les unes par rapport aux autres tandis que le discours didactique se focalise sur cette région préfrontale.

Les graphiques organisationnels permettent ainsi de structurer les connaissances au même titre que les connaissances pouvaient être structurées verbalement pour conduire à un meilleur traitement mémoriel. On comprend alors pourquoi un graphique organisationnel peut servir de structurant antérieur, postérieur ou comparatif sans nécessairement être accompagné d'une séquence verbale correspondante. En tant que structure visuelle de la connaissance, ce type de graphique montre que les connaissances s'organisent de manière hiérarchique, comme c'est le cas de ce diagramme en arbre représentant les différents types de mémoire à long terme (du général au particulier).

Exemple 17. Le cerveau à tous les niveaux (graphique organisationnel à partir d'un diagramme)

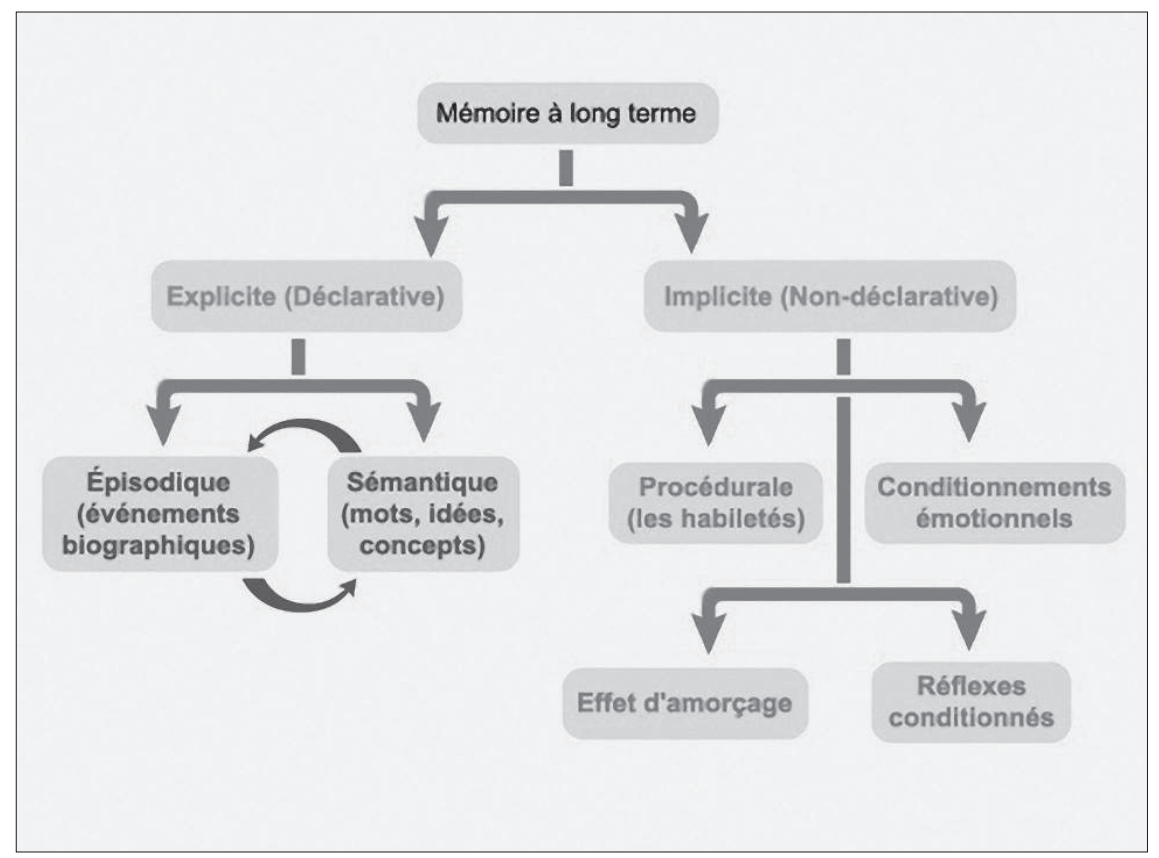

Ce diagramme, qui correspond en fait à une « carte conceptuelle », montre, à travers une présentation spatiale, comment s'organise un ensemble de concepts en mettant en évidence les types de relations qui les unissent. 
J'appelle graphique polyfonctionnel un graphique qui cumule au minimum deux fonctions simultanément. Ainsi, le graphique reformulé d'Atkinson et Shiffrin dans l'e-conférence consacrée au cerveau cumule à lui seul quatre fonctions différentes.

Exemple 18. Le cerveau à tous les niveaux (graphique polyfonctionnel)

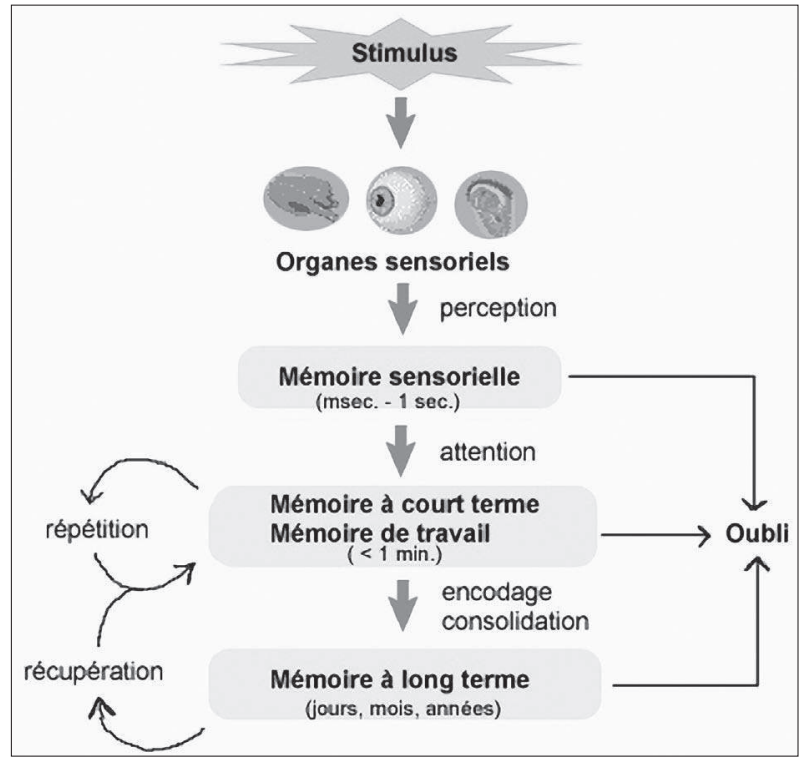

Par sa dimension organisationnelle, ce graphique montre la relation entre un stimulus, les organes sensoriels et les différents types de mémoire; par sa dimension transformationnelle, il montre comment à partir d'un stimulus une perception peut passer de la mémoire sensorielle à la mémoire à long terme dans le temps; par sa dimension relationnelle, le graphique montre des relations quantitatives entre les différentes mémoires (exprimées par le temps); et enfin, par sa dimension interprétative, ce graphique permet de rendre compte d'un phénomène abstrait et invisible qui consiste à montrer la relation entre un stimulus et la mémoire.

Dans la majorité des e-conférences, les stimuli visuels peuvent jouer un rôle primordial, qu'il s'agisse de graphiques ou d'enrichissements typographiques (couleur, graisse, etc.) car, en tant qu'accroches, ils constituent des « passeports » vers la mémoire de travail.

\subsection{Utilisabilité et charge cognitive}

L'utilisabilité d'une e-conférence est également un facteur primordial qui peut favoriser ou non les processus de mémorisation. Concept très répandu dans le monde anglo-saxon, l'utilisabilité réfère à la manière dont 
un usager apprend à utiliser un « produit » pour atteindre ses objectifs et à la manière dont il en tire satisfaction.

En matière de site web, selon Nielsen et Molich (1990) ou Rubin et Chissnell (2008), une bonne utilisabilité doit être facile à apprendre, à mémoriser, à utiliser et produire du plaisir. L'e-conférence consacrée aux «Sciences de la vie au lycée » montre une utilisabilité directe permettant aux registres sensoriels d'appréhender d'un seul regard les contenus de connaissance liés, par exemple, aux régulations physiologiques.

Exemple 19. Les sciences de la vie au lycée (utilisabilité)

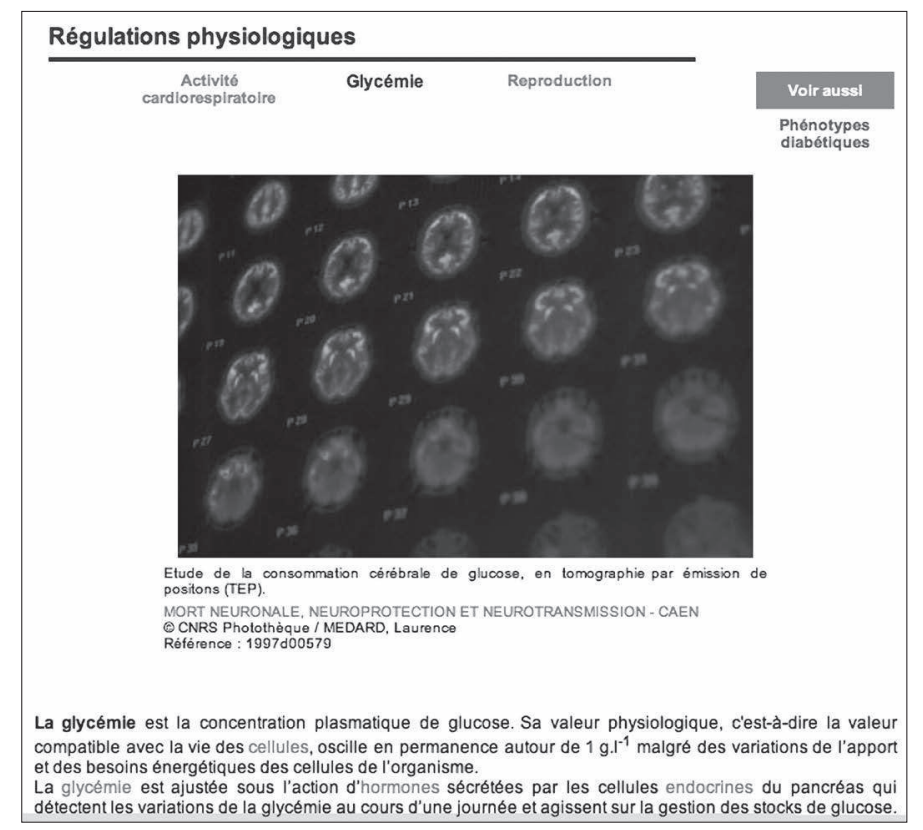

Si le premier contact de l'apprenant avec la rubrique « Régulations physiologiques » nécessite un apprentissage et une compréhension de cette structure tripartite, chaque nouvelle visite ultérieure, pour peu qu'elle soit rapprochée dans le temps, ne visera qu'à renforcer en mémoire cet apprentissage correspondant simultanément à la structure des pages web et des connaissances grâce aux trois entrées hypertextuelles.

Les stimuli mémoriels, qu'il s'agisse ici des graphiques ou des caractéristiques de l'utilisabilité directe, concourent à rendre les connaissances d'une e-conférence plus accessibles encore en exploitant les dimensions visuelles et hypertextuelles du web.

S'intéresser à la question de la mémoire dans les discours de transmission des connaissances, et plus précisément dans les discours didactiques, 
oblige à dépasser la conception traditionnelle que peut avoir l'analyste du discours sur son objet, à savoir que le discours ne serait qu'un paquet d'informations linguistiques. Cela tient à la fois à l'objet d'étude retenu et à la manière dont on envisage le discours didactique.

L'e-conférence, par sa nature et son ancrage sur un support visuel, oblige à dépasser cette conception du discours et à le considérer comme un tout sémiotiquement hétérogène, ce qui contraint l'analyste d'obédience linguistique à se doter de catégories descriptives qu'il emprunte à d'autres domaines, des sciences de l'éducation aux sciences cognitives en passant par le domaine de la communication visuelle.

Une approche par la mémoire, entendue comme lieu de filtrage, d'encodage et de traitement de l'information à partir d'une diversité de stimuli, oblige nécessairement à traquer les stratégies discursives au sens large qui pourraient favoriser ce processus d'intégration d'informations nouvelles en mémoire, correspondant à l'apprentissage de nouvelles connaissances. Le concept de mémoire permettrait de ce fait de renouveler l'angle par lequel on peut analyser les discours didactiques.

On a voulu montrer que l'e-conférence était un genre mémoriel à deux niveaux :

- au sens cognitif du terme d'abord, puisqu'une e-conférence vise à transmettre des connaissances dont on espère qu'elles vont intégrer les réseaux de connaissances existants dans la mémoire à long terme; - au sens social du terme ensuite (Bakhtine 1984, Bronckart 1996), puisqu'une e-conférence est le résultat de pratiques sociales souvent inconscientes dans la mesure où tous ceux qui les conçoivent n'élaborent pas nécessairement leur matériel en tenant compte des paramètres cognitifs de l'apprentissage et qu'il revient donc à l'analyste, à partir d'un corpus d'instances du genre, d'observer les stratégies discursives agissant au niveau des processus de mémorisation comme autant de représentations mentales projetées du genre.

Chacune des stratégies illustrées aurait pu faire l'objet d'une analyse à part entière. Cette perspective ne peut s'envisager qu'à partir du moment où le cadre général qui décrit les rapports du genre à la mémoire est posé, ce qu'on a cherché à faire ici.

Florimond Rakotonoelina

Université Sorbonne Nouvelle - Paris 3

SYLED-CEDISCOR (EA 2290) 


\section{Corpus (consulté en décembre 2009)}

1. "Le cerveau à tous les niveaux " (section " Mémoire et apprentissage ») : http://lecerveau.mcgill.ca/

2. Les sciences de la vie au lycée (section « Régulations physiologiques ») : http://www.cnrs.fr/cnrs-images/sciencesdelavieaulycee/index.htm

3. Webergos, ergonomie des interfaces web (section « L'écriture Web ») : http://www-public.it-sudparis.eu/ milon//index.php

4. L'aventure des écritures (section « Matières et formes ») : http://classes. bnf.fr/dossisup/index.htm

5. Comment écrire un rapport en français (section « Comment situer vos idées et celles des autres? ») : http://www.uniovi.es/ecrire/index.htm

\section{Références}

Ally, M., 2008, « Foundations of educational theory for online learning », dans T. Anderson et F. Elloumi (eds), Theory and pratice of online learning, Athabasca University, p. 3-31.

Anderson, J. R., 2000, Learning and memory : An integrated approach. New York, Wiley.

Atkinson, R. C., et Shiffrin, R. M., 1968, « Human memory : A proposed system and its control processes », dans K. W. Spence et J. T. Spence (eds), Psychology of learning and motivation : Advances in research and theory (vol. 2), New York, Academic Press, p. 89-195.

Ausubel, D. P., 1960, « The use of advance organizers in the learning and retention of meaningful verbal material », Journal of Educational Psychology, $\mathrm{n}^{\circ}$ 51, p. 267-272.

Ausubel, D. P., Novak, J. D., et Hanesian, H., 1978, Educational psychology : A cognitive view, New York, Holt, Rinehart and Winston.

Badre, A., 2002, Shaping web usability : Interaction design in context, Boston, Addison-Wesley.

Bakhtine, M., 1984, Esthétique de la création verbale, Paris, Gallimard, coll. «Bibliothèque des idées ».

Beacco, J.-C., et Moirand, S., 1995, « Autour des discours de transmission de connaissances $»$, Langages, $n^{\circ} 117$, p. 32-53.

Beder, H., 1989, " Purposes and philosophies of adult education », dans S. B. Merriam et P. M. Cunningham (eds), Handbook of adult and continuing education, San Francisco, Jossey-Bass Publishers, p. 37-50. 
Bligh, D. A., 2000, What's the use of lectures?, San Francisco, CA, JosseyBass Publishers.

Braisby, N., et Gellatly, A. (eds), 2005, Cognitive psychology, Milton Keynes, UK, Oxford University Press in association with the Open University.

Bronckart, J.-P., 1996, Sciences des discours : Activité langagière, textes et discours, Lausanne, Delachaux et Niestlé.

Brown, S., et Race, P., 2002, Lecturing : A practical guide, London, Kogan Page.

Bruner, J. S., 1960, The process of education, Cambridge, Harvard University Press.

Carliner, S., 2005, « In defense of the online "lecture" », dans P. Kommers et G. Richards (eds), World conference on educational multimedia, hypermedia and telecommunications 2005, Montreal, Canada, AACE, p. 27-69. —, 2008, « A holistic framework of instructional design for e-learning », dans S. Carliner et P. Shank (eds), The e-learning handbook : Past promises, present challenges, San Francisco, Pfeiffer, p. 307-358.

Clark, R. C., « Four architectures of instruction », Performance Improvement, $\mathrm{n}^{\circ} 39$, p. 31-38.

—, 2002, «Applying cognitive strategies to instructional design », Performance Improvement, vol. 41, $\mathrm{n}^{\circ} 7, \mathrm{p} .8-14$.

Clark, R. C., et Mayer, R. E., 2008, E-Learning and the science of instruction: Proven guidelines for consumers and designers of multimedia learning, San Francisco, CA, Pfeiffer.

Conti, G. J., 2007, « Identifying your educational philosophy : Development of the philosophies held by instructors of lifelong-learners (PHIL) », MPAEA Journal of Adult Education, vol. 36, n 1, p. 19-35.

Doolittle, P. E., 1999, « Constructivism and online education », DOI : 10.1.1.121.4072.

Downing, K. F., et Holtz, J. K., 2008, Online science learning : Best practices and technologies, Hershey, PA, Information Science Reference.

Driscoll, M., et Carliner, S., 2005, Advanced web-based training strategies : Unlocking instructionally sound online learning, San Francisco, CA, Pfeiffer.

Dudai, Y., 2002, Memory from A to Z : Keywords, concepts, and beyond. Oxford, UK, New York, Oxford University Press.

Dumortier, J., 2001, Lisibilité du discours didactique - réflexions sur la compréhension en lecture des différents écrits disciplinaires, Université de Liège, Service de Didactique des Langues et Littératures romanes, http:// www.cifen.ulg.ac.be/agreg/doc/lecture_dumortier.pdf.

Elias, J. L., et Merriam, S. B., 2005 [1980], Philosophical foundations of adult education ( $3^{\mathrm{e}}$ éd.), Malabar, Krieger Publishing Company. 
Ertmer, P. A., et Newby, T. J., 1993, « Behaviorism, cognitivism, constructivism : Comparing critical features from an instructional design perspective », Performance Improvement Quarterly, vol. 6, n ${ }^{\circ} 4$, p. 50-72, DOI : 10.1111/j.1937-8327.1993.tb00605.x.

Farrah, S. J., 1990, « Lecture », dans M. W. Galbraith (ed.), Adult learning methods : A guide for effective instruction, Melbourne, FL, Krieger Publishing Company, p. 161-186.

Goodfellow, R., et Lea, M. R., 2007, Challenging e-learning in the university : A literacies perspective, New York, McGraw-Hill.

Herring, S. C., 2009, « Web content analysis : Expanding the paradigm », dans J. Hunsinger, L. Klastrup, et M. Allen (eds), International handbook of internet research, New York, Springer, p. 233-247.

Hitch, G. J., 2005, « Working memory », dans N. Braisby et A. Gellatly (eds), Cognitive psychology, Milton Keynes, UK, Oxford University Press in association with the Open University, p. 307-341.

Horgan, J., 2003, « Lecturing for learning », dans H. Fry, S. Ketteridge, et S. Marshall (eds), A handbook for teaching \& learning in higher education, London, Sterling, VA, Kogan Page, p. 75-90.

Jacobi, D., 1985, « Sémiotique du discours de vulgarisation scientifique », Semen, 2, http://semen.revues.org/document4291.html

Jacobi, D., 1999, La communication scientifique. Discours, figures, modèles, Grenoble, Presses Universitaires de Grenoble.

Jordan, A., Carlile, O., et Stack, A., 2008, Approaches to learning : A guide for teachers, Maidenhead, McGraw-Hill.

Kintsch, W., Patel, V. L., et Ericsson, K. A., 1999, « The role of long-term working memory in text comprehension », Psychologia, vol. 42, n 4, p. 186-198.

Lawrence, D., et Tavakol, S., 2007, Balanced website design, Londres, Springer-Verlag.

Miller, G. A., 1956, « The magical number seven, plus or minus two : Some limits on our capacity for processing information », Psychological Review, vol. 63, n² 2, p. 81-97, DOI :10.1037/h0043158.

Moirand, S., 1990, « Pour une linguistique du discours adaptée à des objectifs didactiques », Journal of Applied Linguistics, n ${ }^{\circ}$, p. 59-74.

—, 1992, «Autour de la notion de didacticité », Les Carnets du Cediscor, $\mathrm{n}^{\circ} 1$, p. 9-20.

—, 1994, « Présentation », dans S. Moirand, A. Ali Bouacha, J.-C. Beacco, et A. Collinot (dir.), Parcours linguistiques de discours spécialisés, Berne, Peter Lang, p. xiii-xxi.

Nielsen, J., et Molich, R., 1990, « Heuristic evaluation of user interfaces », dans Proceedings of the SIGCHI conference on human factors in computing systems : Empowering people, Seattle, WA, USA, p. 249-256. 
Paivio, A., 1986, Mental representations : A dual coding approach, Oxford, Clarendon Press.

Pavlov, I. P., 1927, Conditioned reflexes : An investigation of the physiological activity of the cerebral cortex, Londres, Oxford University Press, Humphrey Milford.

Petiot, G., 1992, « Hétérogénéités sémiotique et discursive », Les Carnets du Cediscor, $\mathrm{n}^{\circ}$ 1, p. 79-93.

Pritchard, A., 2008, Ways of learning. Learning theories and learning styles in the classroom, New York, Routledge.

Rakotonoelina, F., 1999, « Le cédérom : L’hétérogénéité sémiotique au service de la transmission des savoirs ", dans J.-C. Beacco (dir.), L'astronomie dans les médias. Analyses linguistiques de discours de vulgarisation, Paris, Presses de la Sorbonne Nouvelle, p. 99-138.

—, 2002, «Écriture numérique et révolution des genres? Anamorphose du genre "débat public" ", dans B. Chapelin (dir.), Actes du colloque "écritures en ligne: Pratiques et communautés », Rennes, Université Rennes 2, p. 183-203, http://archivesic.ccsd.cnrs.fr/sic_00126719/fr/.

—, 2008, « De l'in-formation à l'e-formation : transmission des connaissances et plasticité discursive du genre e-conférence », Communication au colloque «Analyse de discours et demande sociale : enjeux théoriques et méthodologiques » (27-29 novembre 2008, Paris), à paraître dans les Actes. —, 2009a, « Écologie et sites web d'(in-)formation : des discours qui vous veulent du bien », Communication au colloque «Les discours écologistes » organisé par Clara Romero et la CRL, 26 septembre 2009, Paris.

—, 2009b, « Transmission des connaissances et conception numérique : Articulation des fonctionnements visuels et verbaux dans le genre e-conférence », Informations, Savoirs, Décisions Et Médiations, n 37, http://isdm. univ-tln.fr/PDF/isdm37/NEDEP_ISDM_Rakotonoelina.pdf

Rubin, J., et Chisnell, D., 2008, Handbook of usability testing : How to plan, design, and conduct effective tests, Indianapolis, IN, John Wiley \& Sons.

Ruther, A., 2005, « Long-Term memory : Encoding to retrieval », dans N. Braisby et A. Gellatly (eds), Cognitive psychology, Milton Keynes, UK, Oxford University Press in association with the Open University, p. 269-306.

Skinner, B. F., 1974, About behaviorism, New York, Knopf.

Sprenger, M., 1999, Learning and memory : The brain in action, Alexandria, Va., USA, Association for Supervision and Curriculum Development.

Spurgeon, L. P., et Moore, G. E., 1997, « The educational philosophies of training and development professors, leaders, and practitioners », The Journal of Technologies Studies, vol. 23, $\mathrm{n}^{\circ}$ 2, http://scholar.lib.vt.edu/ ejournals/JOTS/Summer-Fall-1997/PDF/3-Spurgeon.pdf. 
Swales, J., 1990, Genre analysis : English in academic and research settings, Cambridge, Cambridge University Press.

Thorndike, E. L., 1913, Educational psychology, New York, Teachers College, Columbia University.

Weinstein, C. E., et Acee, T. C., 2008, « Cognitive view of learning », dans N. J. Salkind et K. Rasmussen (eds), Encyclopedia of educational psychology, Thousand Oaks, CA, Sage Publications, p. 164-165.

Yates, S., et Sumner, T., 1997, «Digital genres and the new burden of fixity », Proceedings of the Hawaiian International Conference on System Sciences, vol. 4, no 30, p. 3-12, http://www2.computer.org/portal/web/csdl/home.

Zinn, L. M., 1990, « Identifying your philosophical orientation », dans M. W. Galbraith (ed.), Adult learning methods, Malabar, FL, Krieger Publishing Company, p. 39-77. 